\title{
A IMPORTÂNCIA DO USO DE PROTETORES SOLARES NA PREVENÇÃO DO FOTOENVELHECIMENTO E CÂNCER DE PELE
}

\section{SILVA, André L. Araújo*; SOUSA, Katya R. Ferreira; SILVA, Aline F.; FERNANDES, Amanda B. Fernandes; MATIAS, Vanessa L.; COLARES, Aracélio V.}

Faculdade Leão Sampaio - Juazeiro do Norte (CE), Brasil.

Recebido em: 24/02/2015; Aceito: 22/04/2015; Publicado: 25/08/2015

\section{RESUMO}

Nas últimas décadas a incidência dos raios ultravioleta aumentou significativamente. Em 2012 eram 1,3 milhões de mortos segundo a Organização Panamericana de Saúde, registrando a segunda maior causa de morte nas Américas. Além do câncer, a incidência solar é a maior causa do fotoenvelhecimento. Na pele causa rugas, perda da rigidez e possibilitando ainda mais o câncer. Assim, o uso de fotoprotetores é de extrema importância para a precaução desses males ainda sem uma cura estabelecida. Foram distribuídos questionários para estudantes universitários de uma instituição de nível superior. A pesquisa aconteceu durante o período de agosto a outubro de 2014. Aplicou-se 283 questionários. Cerca de $36 \%$ dos entrevistados foram do sexo masculino e $64 \%$ do sexo feminino. Aproximadamente $48 \%$ usam fotoprotetores solares com uma frequência 52\%. $85 \%$ declaram que os fotoprotetores tem uma ação importante, enquanto $15 \%$ discordam. Cerca de 97\% recomendariam o uso de fotoprotetores, apenas 3\% dizem que não recomendariam. Enquanto que 31\% afirmam que somente os fotoprotetores já são suficientes para prevenir o câncer, 69\% declararam que não. Mesmo quando 85\% deles sabem da importância do uso e $97 \%$ recomendariam o uso por terceiros, somente $52 \%$ fazem uso dos protetores. Apenas $31 \%$ dizem que somente os protetores já são suficientes na prevenção do câncer. Assim, este trabalho reúne informações importantes sobre o uso dos protetores solares e sobre os níveis de informações de estudantes do ensino superior.

Palavras-chave: Ultravioleta, Fotoprotetores, Fotoenvelhecimento, Pele

\begin{abstract}
In recent decades the incidence of ultraviolet rays increased significantly. In 2012 were 1.3 million dead according to the Pan American Health Organization, recording the second leading cause of death in the Americas. In addition to cancer, the sunlight is a major cause of photoaging. In the skin causes wrinkles, loss of rigidity and allowing even more cancer. Thus, the use of sunscreen is very important to caution these evils still without an established cure. Questionnaires were distributed to university students in a postsecondary institution. The search took place during the period August to October 2014. Was applied to 283 questionnaires. About $36 \%$ of respondents Were do male and $64 \%$ female. About $48 \%$ use solar sunscreens with a frequency of $52 \% .85 \%$ state that the sunscreen has an important action, while $15 \%$ disagree. About $97 \%$ would recommend the use of sunscreens, only 3\% say they do not recommend. While $31 \%$ say that only sunscreens are sufficient to prevent cancer, $69 \%$ said no. Even when $85 \%$ of them know the importance of using and $97 \%$ would recommend the use by third parties, only $52 \%$ use of shields. Only $31 \%$ say that only the guards are sufficient in preventing cancer. This work gathers important information about the use of sunscreens, and information about the levels of higher education students.
\end{abstract}

Keywords: Sunscreens, Ultraviolet, Photoaging, Skin.

*André Luiz de Araújo Silva; Rua Jacinto Rocha, 32. Santa Tereza, Juazeiro do Norte, Ceará. CEP: 63050-450

E-mail: andre luiz $901 @$ live.com Telefone: (88) 988176899 


\section{INTRODUÇÃO}

Nos últimos tempos a radiação aumentou consideravelmente, o que tornou muito importante a difusão do uso dos protetores solares para prevenir doenças de pele principalmente o câncer de pele. Os filtros solares ou protetores solares são substâncias que aplicadas sobre a pele protegem a mesma contra a ação dos raios ultravioletas do sol (ARAÚJO, 2008).

$\mathrm{O}$ espectro solar que atinge a terra é quase completamente de radiação ultravioleta em torno de 100-400nm, menor que o espectro visível (400$800 \mathrm{~nm}$ ) e infravermelho (mais de $800 \mathrm{~nm}$ ). Nosso corpo responde à incidência das radiações de formas diferentes, com calor para as ondas infravermelhas e reações fotoquímicas para as radiações ultravioletas (UV). Essas reações podem ir desde o estímulo de produção de melanina, até leves queimaduras e mutações no DNA que tem sido mais frequentes nos últimos anos (FLOR; DAVOLOS e CORREA, 2006).

A radiação ultravioleta é dividida em UVA, UVB e UVC. A radiação UVC tem menor comprimento de onda e causa efeitos carcinogênicos e mutagênicos. A radiação UVB causa queimaduras, lesões, bolhas e câncer de pele. A radiação UVA tem comprimento de onda maior e é mais lesiva, pois penetra mais profundo à derme $\mathrm{e}$ gera radicais livres, causando envelhecimento.

Dados do Instituto Nacional de Pesquisas Espaciais (INPE) mostram que o Índice Ultravioleta (IUV) da maioria das capitais brasileiras encontrase em níveis considerados Muito Altos ou Extremos, em Fortaleza e na maioria do Nordeste chega a se consultar IUV em 12 quase diariamente, outras capitais como Porto Alegre constam IUV em 8 ou mais. Somente essa informação já seria o suficiente para motivar a população brasileira ao uso de protetores solares e barreiras físicas contra o sol.

Davolos (2006), diz que a necessidade do uso de protetores solares, também denominados fotoprotetores, é uma realidade indiscutível estimase que em 1992 o mercado nacional de protetores solares tenha comercializado 650 toneladas de produtos. Para Lonni (2008), filtros solares são substâncias que, quando adicionadas aos produtos para proteção solar, tem a finalidade de filtrar raios ultravioletas visando proteger a pele de efeitos dos causados por estes raios, como envelhecimento precoce da pele e cânceres.

O Câncer já representava em 2012 a segunda maior causa de morte nas américas, com 2,8 milhões de casos novos e 1,3 milhões de mortos segundo a Organização Panamericana de Saúde (OPS, 2012). E esperam-se 98.420 casos novos de câncer de pele não melanoma nos homens e 83.710 nas mulheres no Brasil, em 2014, dados do Instituto Nacional do Câncer.

Este artigo agrega informações sobre a importância do uso de protetores solares, com relação à prevenção do fotoenvelhecimento e o câncer de pele. Além disso, buscam-se informações importantes sobre o conhecimento entre estudantes da área da saúde e a importância da manutenção de uma boa política preventiva de saúde acerca os danos lesivos do sol.

\section{REFERENCIAL TEÓRICO}

\subsection{RADIAÇÃO}

Considerando-se os hábitos da população em geral, observa-se que grande parte das pessoas se submete de maneira excessiva às radiações solares quase que diariamente (SOUZA, 2008).

$\mathrm{O}$ espectro solar que atinge a superfície terrestre é formado predominantemente por radiações ultravioletas $(100-400 \mathrm{~nm})$, visíveis 
(400-800 nm) e infravermelhas (acima de $800 \mathrm{~nm}$ ). Nosso organismo percebe a presença destas radiações do espectro solar de diferentes formas (DAVOLOS, 2007).

A intensidade da radiação e o comprimento de onda da luz solar dependem de fatores como altitude, latitude, estação do ano, condições atmosféricas e horários. As radiações mais lesivas ocorrem entre as 10-11 horas da manhã e as menos lesivas entre as 16-17 horas da tarde e pode ser particularmente útil observar a regra da sombra, uma vez que as variáveis mencionadas acima não são fixas (PETRI, 2005).

\subsubsection{Tipos de radiação}

Entende-se por radiação ultravioleta, a região do espectro eletromagnético emitido pelo sol, compreendida entre os comprimentos de ondas de 200 a $400 \mathrm{~nm}$ que, por sua vez, subdividem se em três: ultravioleta C ou UVC (200-290 nm), que é dispersa na atmosfera e não chega à superfície terrestre; ultravioleta B ou UVB (290-320nm); ultravioleta A (320-400 nm). Este último ainda pode ser subdividido em UVA-I (320-340nm) e UVA-II (340-400 nm) (LONNI, 2008).

As radiações UVA promovem o bronzeamento direto e são responsáveis pelo fotoenvelhecimento, produção de radicais livres e pela melanogênese (SOUZA, 2004).

\subsection{FOTOENVELHECIMENTO}

O processo de envelhecimento da pele é um fenômeno biológico, que pode ser classificado em 2 componentes: envelhecimento intrínseco e extrínseco (TZAPHLIDOU, 2004).

Como o nome implica, envelhecimento intrínseco é devido à senescências geneticamente controladas, enquanto o envelhecimento extrínseco é devido a fatores ambientais super impostos no envelhecimento intrínseco. Fatores ambientais conhecidos na aceleração de envelhecimento extrínseco são exposições ao sol (fotoenvelhecimento) e cigarros (DRAELOS, 1999).

Pele extrinsecamente envelhecida e exposta ao sol aparece clinicamente como manchada, espessa, amarelada, frouxa, áspera e dura (JENKINS, 2002).

Os tecidos gradualmente passam por mudanças de acordo com a idade, sendo que, na pele, essas alterações são mais facilmente reconhecidas. Observa-se na pele envelhecida ressecamento associado a uma sensação tátil de rugosidade, atrofia, perda de firmeza, pigmentação desigual e lesões proliferativas, sendo esse quadro clínico acelerado na exposição solar (ORIÁ et al., 2003).

Já o fotoenvelhecimento é mais agressivo à superfície da pele, sendo o responsável por modificações como rugas, manchas e o próprio câncer de pele (STEINER, 2004). Sabe-se que o número de rugas na pele tem uma relação direta com a exposição diária ao sol durante a vida (MONTAGNER e COSTA, 2009).

\subsection{CÂNCER DE PELE}

A doença é um crescimento anormal e descontrolado das células que compõem a pele. Estas células se dispõem formando camadas e, de acordo com a camada afetada, definimos os diferentes tipos de câncer. A radiação ultravioleta é a principal responsável pelo desenvolvimento de tumores cutâneos, e a maioria dos casos está associada à exposição excessiva ao sol ou ao uso de câmaras de bronzeamento (SBD, 2012).

A incidência do câncer da pele tem aumentado em todo o mundo nas últimas três décadas sendo essa a forma de câncer mais comum. 
Inúmeras causas têm sido apontadas: mudanças dos hábitos de vida com exposição solar excessiva rarefação da camada de ozônio envelhecimento populacional diagnóstico precoce desses cânceres (HORA, 2003).

O câncer de pele costuma apresentar-se sob três principais formas: melanoma, carcinoma basocelular e carcinoma espinocelular (ou epidermoide). Os carcinomas basocelular e epidermoide são também conhecidos como câncer de pele não melanoma, tipo mais frequentes de câncer de pele e câncer mais frequente na população de pele clara. No Brasil, o câncer de pele não melanoma é o tumor mais incidente em ambos os sexos (SILVA, 2014). Esperam-se 98.420 casos novos de câncer de pele não melanoma nos homens e 83.710 nas mulheres no Brasil, em 2014, segundo o Instituto Nacional do Câncer.

\subsubsection{Tratamento}

Os produtos cosméticos de tratamento devem se adequar às condições anatômicas e fisiológicas da pele, em suas variações individuais. Os cuidados com a integridade funcional, além da anatômica, permitem intervir eficazmente com o objetivo de obter melhoras estéticas da pele (BENY, 2000).

É cada vez maior o interesse das pessoas por uma pele jovem, isenta de rugas e manchas, o que tem estimulado os pesquisadores da área cosmética na busca de se conhecer os mecanismos responsáveis, bem como as principais alterações morfo-histológicas causadas durante o processo de envelhecimento cutâneo (HATZIS, 2004).

Todo e qualquer câncer têm de ser tratados previamente, mesmo os menos letais, evitando o sofrimento do paciente. Felizmente, existem várias opções terapêuticas para o câncer de pele nãomelanoma, como cirurgia excisional, terapia fototerapêutica, cirurgia a laser etc (SBD, 2012). Porém, mesmo com avanços tecnológicos e promessas de cosméticos, a melhor forma de combater o fotoenvelhecimento e o câncer é o prevenção, com o uso de barreiras físicas e conscientização de protetores solares diários.

\section{METODOLOGIA}

Foram distribuídos questionários aos estudantes universitários do curso de Biomedicina da Faculdade Leão Sampaio. A pesquisa aconteceu durante o período de agosto a outubro de 2014. Os desenvolvedores do trabalho aplicaram 310 questionários, nos períodos diurno e noturno. Foram respondidos 283 questionários.

O questionário foi elaborado pelos autores do trabalho e composto de cinco perguntas que avaliam o perfil básico dos entrevistados e seus conhecimentos acerca do tema. 1 - Sexo, 2 - Uso de fotoprotetores, 3 - Importância dos fotoprotetores, 4 e 5 - Conhecimento acerca dos fotoprotetores. No topo do questionário havia a descrição do tema.

A amostra foi determinada através de cálculos estatísticos levando em consideração o número de alunos matriculados no Campi da Faculdade, informação cedida pela coordenação do curso. A população foi composta de 658 alunos, chegando a uma amostra de 310 entrevistas, levando em consideração hipóteses de negação de resposta, ou extravios do material, como marcações erradas no questionário. Os dados foram computadorizados e sua análise estatística foi realizada de forma descritiva com frequências analíticas (n) e absolutas (\%).

\section{RESULTADOS E DISCUSSÃO}

Foram respondidos 283 questionários, onde $102(36 \%)$ do sexo masculino e $183(64 \%)$ do 
sexo feminino. 137 (48\%) usam fotoprotetores solares com frequência e 146 (52\%) responderam que não. $240(85 \%)$ declaram que os fotoprotetores têm uma ação importante, enquanto outros 43 (15\%) discordam. Um elevado número de 274 (97\%) recomenda como futuros biomédicos o uso de fotoprotetores, apenas $9(3 \%)$ dizem que não recomendariam. E por último, $89(31 \%)$ afirma que somente os fotoprotetores já são suficientes para prevenir o câncer, 194 (69\%) declararam que não.

$\mathrm{O}$ alto índice de radiação solar é um sério problema, e muitas pessoas encontram-se desinformadas acerca dos efeitos deletérios causados por exposição intensa. Mesmo com a frequente assolação de câncer de pele no país, a proteção e prevenção com a exposição é ainda pouco preconizada (SBD, 2006).

É necessário que se faça o uso consciente dos fotoprotetores e de maneira correta, como aplicar 20 a 30 minutos antes da exposição, repassar a cada 3 horas, não excluir o uso em dias nublados e utilizar filtros UVA e UVB (CABRAL; PEREIRA e PARTATA, 2013).

Olhando por um ângulo financeiro, o custo de um tratamento total para o câncer de pele em média por paciente chega até 2700 reais, dependendo do estágio de descoberta e tipo de câncer. Estes valores podem gerar um impacto de 37 milhões de reais ao sistema público de saúde (SOUZA et al., 2011).

Este trabalho avaliou estudantes universitários do curso de Biomedicina de Juazeiro do Norte em 2014. Foram constatados que a maioria dos participantes do estudo são mulheres (64\%), como já era esperado, pois o curso contém mais mulheres matriculadas.

O curso se dividiu ao responder sobre o uso dos fotoprotetores, pois $52 \%$ ainda responderam que sim, um número bastante baixo quanto ao esperado, haja visto que um estudo de em porto alegre demonstrou que $82,5 \%$ dos universitários utilizavam protetores solares (COSTA e WEBER, 2004) na região metropolitana de Porto Alegre. Já na nossa região esse número deveria aumentar, pois estamos em uma região mais acometida pelo calor do país.

A grande maioria (85\%) dos entrevistados afirma saber da importância da ação dos fotoprotetores. Um número relevante e deveras contraditório, pois uma parte dessa amostra mesmo sabendo da importância escolhe não usar os protetores solares. Outro número interessante é quando os entrevistados em quase unanimidade (97\%) recomendam o uso dos fotoprotetores, mesmo sem essa totalidade utilizar esse mecanismo de proteção. De fato, os fotoprotetores são conhecidos pelos universitários e até recomendados entre eles, porém não é feito o mais importante, que é o uso deste meio protetor.

Dos entrevistados, 89 (31\%) consideram que somente os fotoprotetores já são suficientes para o combate ao câncer de pele e o fotoenvelhecimento. Isto demonstra que outros meios de proteção, como barreiras físicas estão sendo esquecidas e não praticadas como defesa. $\mathrm{O}$ uso de camisetas, bonés e guarda-sol é de extrema importância, e o uso de ambas as medidas protetoras são cumulativas. Ou seja, o uso tanto de fotoprotetores como de barreiras físicas potencializa o efeito de defesa do organismo.

Estes dados servem para obter 0 conhecimento do nível de uso dos fotoprotetores e a propagação da informação dos mesmos, assim podendo de melhor maneira enfatizar a orientação individual e coletiva dos hábitos corretos. Este trabalho ressalta a importância desta orientação específica para o câncer advindo dos raios UV através da socialização parental, incentivo escolar, orientação por órgãos públicos em mídias entre outros modelos de propagação de informação. 


\section{CONCLUSÃO}

No trabalho foram avaliados adultos jovens de uma Faculdade na cidade de Juazeiro do Norte em 2014 entre agosto e outubro, referente ao uso de fotoprotetores solares na prevenção do fotoenvelhecimento e câncer. Apenas metade dos universitários faz uso dos fotoprotetores solares apesar de conhecer sua importância. Uma parte ainda afirma que somente os fotoprotetores são suficientes para a prevenção, ignorando o uso de barreiras físicas.

\section{REFERÊNCIAS}

ARAUJO, T. S. E.; SOUZA S. O. Protetores Solares e os efeitos da radiação Ultravoleta. Scientia Plena, São Cristovão, v. 4, n. 11, Ago. 2008.

<http://maquel.com.br/media/academico/artigo/01_ 01_08_protetoresearadia_c_eosolar.pdf $>$. Data de acesso:04 de Fevereiro de 2015.

CABRAL, L.D.S.; PEREIRA, S.O.; PARTATA, A.K. Filtros solares e fotoprotetores - uma revisão. Infarma, Araguaia- TO, Volume 25, Número 02. 2013.

http://revistas.cff.org.br/?journal=infarma\&page $=$ ar ticle\&op=view\&path\%5B\%5D=447\&path\%5B\%5 $\underline{\mathrm{D}=433}$

COSTA, F. B.; WEBER, M. B. Avaliação dos hábitos de exposição ao sol e de fotoproteção de universitários da região metropolitana de Porto Alegre, RS. Investigação Clínica, Laboratorial e Terapêutica, Rio de Janeiro, Mar/Abr 2004. 149155.

CHORILLI, M. Abordagens no estudo do envelhecimento cutâneo em diferentes etnias.

DRAELOS, Z.D. Cosméticos em dermatologia. 2.ed. Rio de Janeiro: Editora Revinter, 1999.
FlOR, J.; DAVOLOS, M. R.; CORREA, M. A. Protetores Solares, Agosto 2006. Disponivel em: <http://www.scielo.br/pdf/qn/v30n1/26.pdf>. Acesso em: 25 Setembro 2014.

GIL, A. C. Métodos e Técnicas de Pesquisa Social. 6 ed, São Paulo: Atlas, 2008

HATZIS, J. The wrinkle and its measurement - a skin surface profilometric method. Micron 2004 (35): 201.219 .

BENY, M. G. Fisiologia da pele. Cosmetics \& Toiletries. 2000 (12): 44-50.

HATZIS, J. The wrinkle and its measurement - a skin surface profilometric method. Micron. 2004 (35): 201.219.

MONTAGNER, S.; COSTA, A. Bases biomoleculares do fotoenvelhecimento. Anais Brasileiros de Dermatologia, Rio de Janeiro, Julho 2009.

OPS. Organização Panamericana de Saúde, 2014. Disponível em: <http://www.paho.org/hq/index.php?option=com_c ontent $\&$ view $=$ article $\& i d=292 \% 3$ Acancer $\&$ catid $=18$ 66\%3Anmh-cancer-home \&Itemid=386\&lang=es $>$. Acesso em: 17 Outubro 2014.

SBD, Sociedade Brasileira de Dermatologia. Análise de dados das campanhas de prevenção ao câncer da pele promovidas pela Sociedade Brasileira de Dermatologia de 1999 a 2005. Anais Brasileiros de Dermatologia, Rio de Janeiro, v. 81 n. $\quad 06, \quad$ Nov./Dec. 2006 http://www.scielo.br/scielo.php?script=sci_arttext\& pid=S0365-05962006000600004\&lang=pt

SBD. Sociedade Brasileira de Dermatologia. 2012. Disponivel em: <http://www.sbd.org.br/>. Acesso em: 15 Outubro 2014.

SILVA, J. A. G. D. Estimativa 2014 - Incidência de Câncer no Brasil. Rio de Janeiro: [s.n.], 2014.

SOUZA R. J. S. P., et al. Estimativa do custo do tratamento do câncer de pele tipo não-melanoma no Estado de São Paulo - Brasil. Anais Brasileiros de Dermatologia. Rio de Janeiro, v. 86, n. 04, 
Jul./Ago. 2011. Disponível em: $<$ http://www.scielo.br/scielo.php?script=sci_arttext \&pid=S0365-05962011000400005\&lang=pt $>$

STEINER, D. As Influências raciais e a pele. Cosmetics \& Toiletries 1996 (8): 28-30.

Oriá, R.B.; SANTANA, E.N.; FERNANDES, M.R.; FERREIRA, F.V.A.; BRITO, G.A .C. Estudo das alterações relacionadas com a idade na pele humana, utilizando métodos de histo-morfometria e autofluorescência. Anais Brasileiros de Dermatologia 2003 78(4): 25-434.

TZAPHLIDOU, M. The role of collagen and elastin in aged skin: an image processing approach. Elsevier 2004 (35): 173-177. 